\title{
Loudspeaker configuration in reverberation rooms for sound absorption measurement using room mode determination.
}

Moos, Niels Peter; Jeong, Cheol-Ho; Bolberg, Mads

Published in:

Proceedings of inter-noise 2021

Publication date:

2021

Document Version

Peer reviewed version

Link back to DTU Orbit

Citation (APA):

Moos, N. P., Jeong, C-H., \& Bolberg, M. (2021). Loudspeaker configuration in reverberation rooms for sound absorption measurement using room mode determination. In Proceedings of inter-noise 2021

\section{General rights}

Copyright and moral rights for the publications made accessible in the public portal are retained by the authors and/or other copyright owners and it is a condition of accessing publications that users recognise and abide by the legal requirements associated with these rights.

- Users may download and print one copy of any publication from the public portal for the purpose of private study or research.

- You may not further distribute the material or use it for any profit-making activity or commercial gain

- You may freely distribute the URL identifying the publication in the public portal

If you believe that this document breaches copyright please contact us providing details, and we will remove access to the work immediately and investigate your claim. 


\title{
Loudspeaker configuration in reverberation rooms for sound absorption measurement using room mode determination
}

\author{
Niels Peter Moos ${ }^{1}$ \\ Acoustic Technology, Electrical Engineering Department, Technical University of Denmark \\ Ørsteds Plads, Building 352, 2800 Kgs. Lyngby, Denmark \\ Cheol-Ho Jeong ${ }^{2}$ \\ Acoustic Technology, Electrical Engineering Department, Technical University of Denmark \\ Ørsteds Plads, Building 352, 2800 Kgs. Lyngby, Denmark \\ Mads Bolberg ${ }^{3}$ \\ ROCKWOOL International A/S \\ Hovedgaden 584, 2640 Hedehusene, Denmark
}

\begin{abstract}
For statistical sound absorption measurements in reverberation rooms, it is preferable to excite as many room modes as possible. But the number of excited room modes is significantly affected by many factors, among which one dominating factor is the loudspeaker location particularly at frequencies below the Schroeder frequency. In turn, this could significantly influence the statistical absorption coefficient obtained according to ISO 354 . Therefore, this study aims to investigate the impact of loudspeaker configurations on the excitation of room modes. Frequency response functions are measured using a broad band steady state noise signal using four sound sources at various locations, and the numbers of excited room modes in the one-third octave bands are quantified and compared against those by Green's function simulations. A procedure for determining favorable loudspeaker positions based on the excited room modes is proposed, which can be a useful input to the working group of the ISO 354 standard.
\end{abstract}

\section{INTRODUCTION}

The location of a loudspeaker during a sound absorption measurement, can impact the result of the frequency response function. In theory, when a loudspeaker is located in the corner of a room the highest number of room modes will be excited [1]. However, corner loudspeakers are not commercially available at this moment. On the other hand omnidirectional loudspeakers are a key part of many acoustical laboratories. Although the result of the frequency response function is also

\footnotetext{
${ }^{1}$ nielspetermoos@yahoo.dk

${ }^{2}$ chj@elektro.dtu.dk

${ }^{3}$ mads.bolberg@rockwool.com
} 
impacted by the receiver position, this study is focused on the position of the loudspeaker, as multiple microphone positions are used for a sound absorption measurement.

Sound absorption measurements are performed in reverberation chambers with different designs around the world. Although a guideline is set for theses measurements, different results may be achieved due to poor inter laboratory reproducibility [2,3]. Therefor this study aims to suggest a method for choosing preferable loudspeaker locations in reverberation chambers.

As the omnidirectional loudspeakers are not necessarily placed in corners but at nodal points, they may cancel some room modes and thereby impact the sound absorption measurement. Thus it is important to place the loudspeaker at a location at which most possible room modes are excited.

\section{METHODOLOGY}

\subsection{Greens function simulations}

To simulate the frequency response functions for rectangular reverberation chambers, Green's function is used. It is written as follows: [4]

$$
G\left(\mathbf{r}, \mathbf{r}_{\mathbf{0}}\right)=-\frac{1}{V} \sum_{m} \frac{\psi_{m}(\mathbf{r}) \cdot \psi_{m}\left(\mathbf{r}_{\mathbf{0}}\right)}{k^{2}-k_{m}^{2}-\frac{j k}{\tau_{m} c}}
$$

where $\tau_{m}$ is the time constant of the $n$ 'th mode and $c$ is the speed of sound. $\psi_{m}$ denotes the sound pressure at the sound source $\mathbf{r}$ and receiver $\mathbf{r}_{\mathbf{0}}: 2$

$$
\psi_{N}(x, y, z)=\sqrt{\varepsilon_{n_{x}} \cdot \varepsilon_{n_{y}} \cdot \varepsilon_{n_{z}}} \cos \left(\frac{n_{x} \pi x}{l_{x}}\right) \cos \left(\frac{n_{y} \pi y}{l_{y}}\right) \cos \left(\frac{n_{z} \pi z}{l_{z}}\right)
$$

where $\sqrt{\varepsilon_{n_{x}} \cdot \varepsilon_{n_{y}} \cdot \varepsilon_{n_{z}}}$ is the normalization factor, where $\varepsilon_{0}=1$ and $\varepsilon_{m}=2$ for $m>0$. The simulations are only valid for a rectangular room without diffusing elements. Figure 1 shows an example of the frequency response function result of Green's function which is used to compare against the function of the measurement.

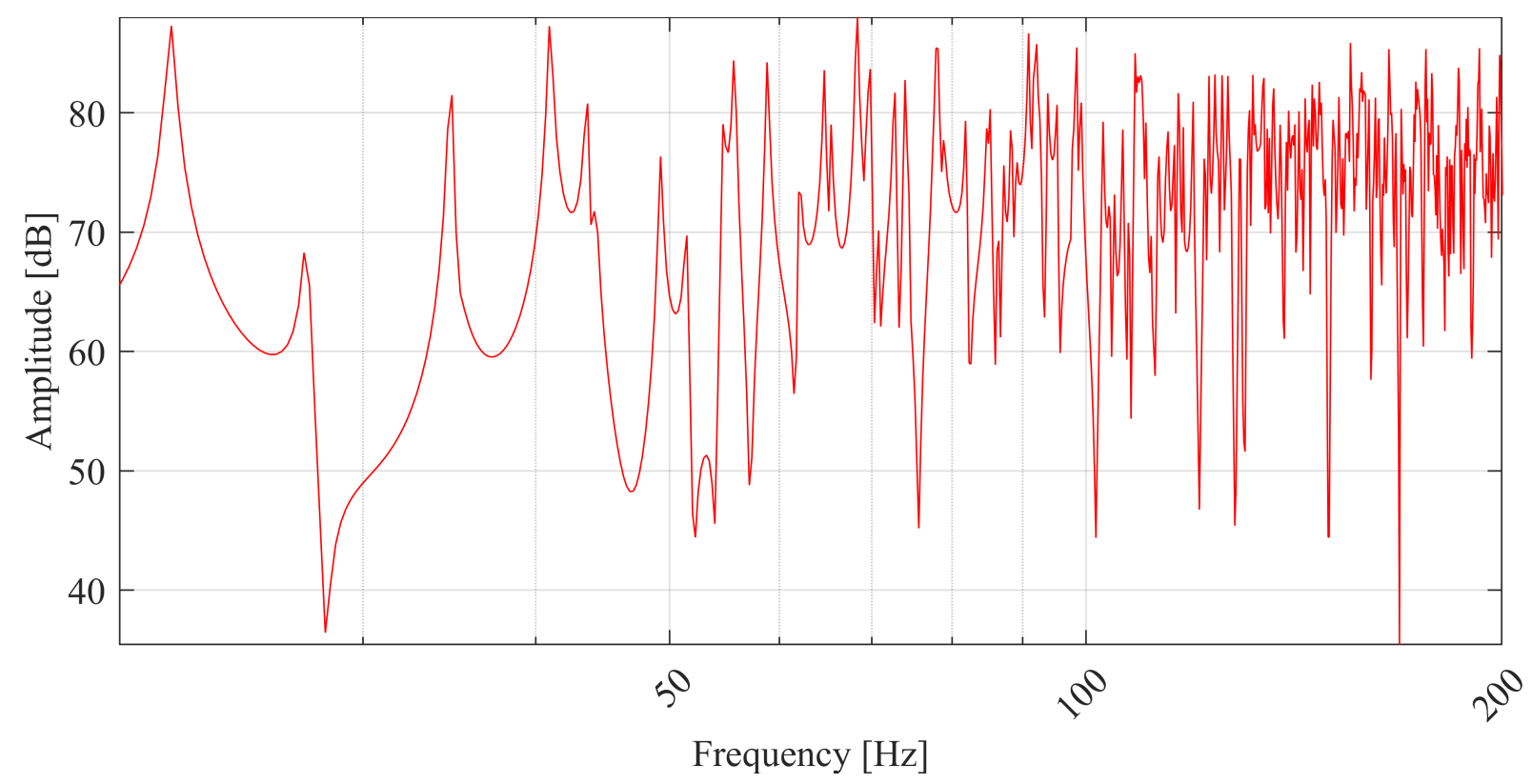

Figure 1: Example of frequency response function from Green's function in a chamber with dimensions $7.85 m \times 6.26 m \times 4.93 m$. The sound source is located in a corner at $(7.85,6.26,0.00)$, and the receiver at an edge at $(0.05,3.45,0.25)$. 
Figure 1 is analyzed to find the peaks which are all assumed to be room mode excitations. It is not considered a requirement that the Green's functions peaks are at the same frequency as those of the measurements, but it is expected that they will look alike and have a large percentage of similar peaks.

\subsection{Peak determination simulations}

When modal overlap is sufficiently low, a peak in sound pressure level (SPL) can be considered a room mode excitation. For this reason the procedure is not considered applicable above the Schroeder frequency, at which the modal overlap exceeds three. Not all peaks can be considered a room mode, as the frequency response function does not result in a smooth curve from the measurement. To take this into account, a procedure is proposed to locate the peaks and sort out most of the peaks which are not to be considered a room mode excitation. The first part of the method, is to determine the energy average of the peaks in intervals of $10 \mathrm{~Hz}$, then removing any peaks below the average from the count. This procedure is used first, to locate local peaks. Following this, the energy average of the curve is determined in $1 / 3$ octave bands and peaks left in the count below this average is removed. This procedure ensures that any peaks which excitations are too low in the $1 / 3$ octave bands are removed. The energy average is determined as in Equation 3 [1]:

$$
\bar{L}=10 \log _{10} \cdot\left(\frac{1}{n} \cdot\left(10^{L_{1} / 10}+10^{L_{2} / 10}+\cdots+10^{L_{n} / 10}\right)\right) \mathrm{dB}
$$

where $n$ is the number of data points in the interval and $L_{n}$ is the measured sound pressure levels. Following the peak removal, the peaks are counted, analyzed and compared with other positions to determine which position is most favorable. An example showing the procedure of peak removal is shown in Figure 2.

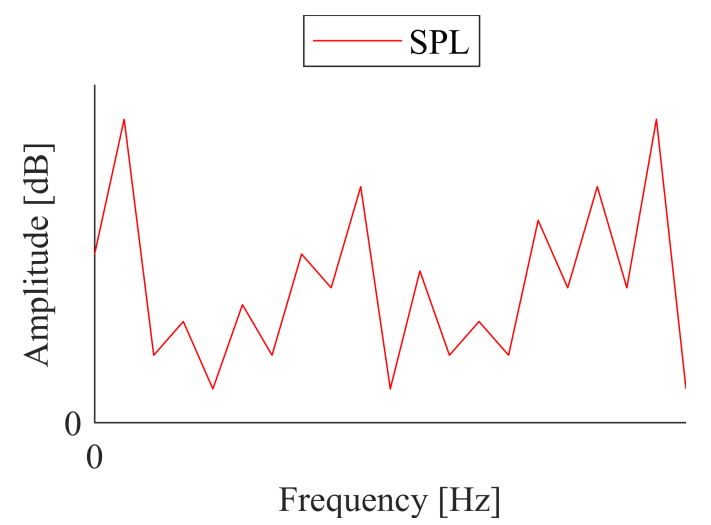

(a)

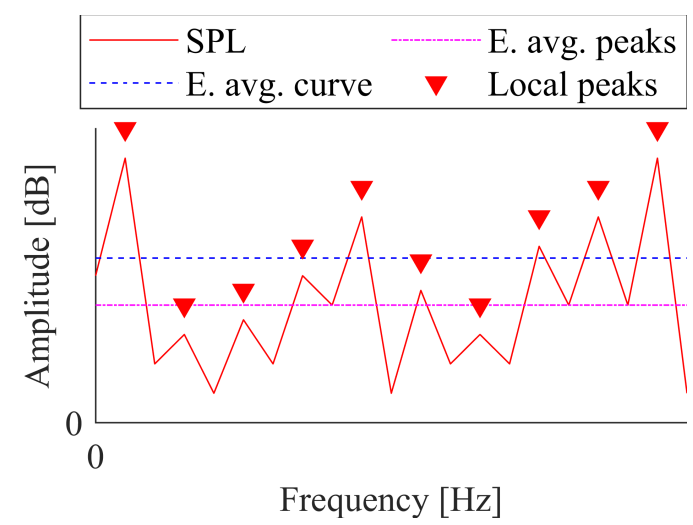

(b)

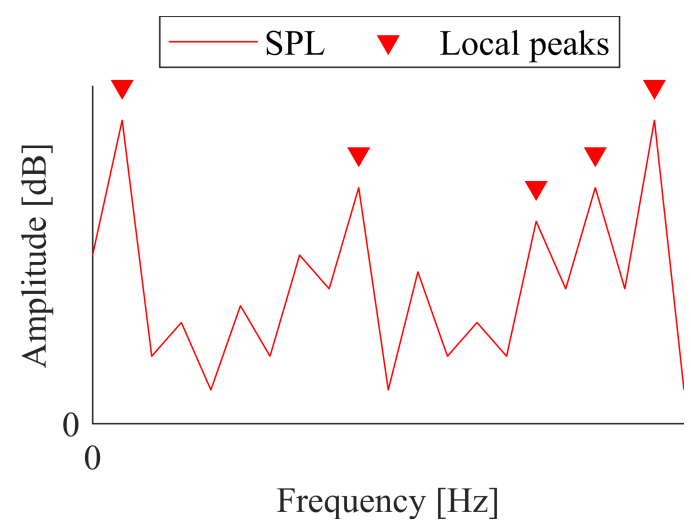

(c)

Figure 2: Example of peak removal procedure 
The method shown in Figure 2 from left to right begin with the measured data. On the second graph, the peaks are located and the energy averages are determined and displayed. On the final frame the measurement is now displayed with only peaks above the energy average included in the count.

\subsection{Measurements}

To obtain data, measurements were conducted in a reverberation chamber by using an omnidirectional loudspeaker as well as a corner loudspeaker. The corner loudspeaker is DTU-made with a membrane of $25 \mathrm{~cm}$ in diameter. The measurements were conducted in a rectangular reverberation chamber, such that the data would be comparable with the results of the Green's function simulation. The reverberation chambers can be seen in Figure 3.

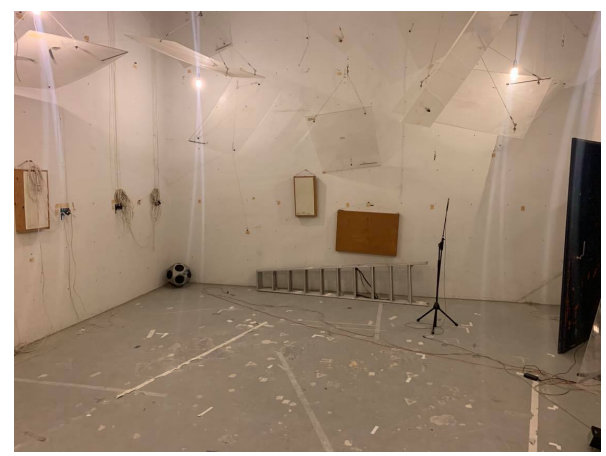

(a)

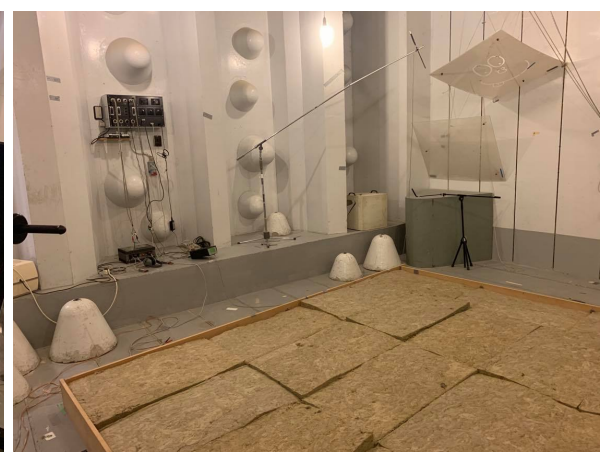

(b)

Figure 3: Pictures from inside reverberation chamber 904 in subfigure (a) and 005 in subfigure (b)

Although the chambers have approximately the same outer dimensions, they have different volumes. The outer dimensions of both chambers are roughly $7.85 m \times 6.26 m \times 4.93 m$. From Figure $3 \mathrm{~b}$ it is possible to see the complex shape of chamber 005 making its volume $215 \mathrm{~m}^{3}$ as opposed to chamber 904's $242 \mathrm{~m}^{3}$. For further specifics on the measurements and chambers see [5].

\subsection{Choice of omnidirectional loudspeaker}

The frequency response function of three omnidirectional loudspeakers were studied in an anechoic chamber resulting in Figure 4 from [5]. The measurement was conducted in an anechoic chamber, using four microphone positions around the loudspeakers each with $1 \mathrm{~m}$ distance to the loudspeaker. The energy average of the four resulting frequency response curves is calculated and shown in Figure 4. For further information on the method and equipment see [5].

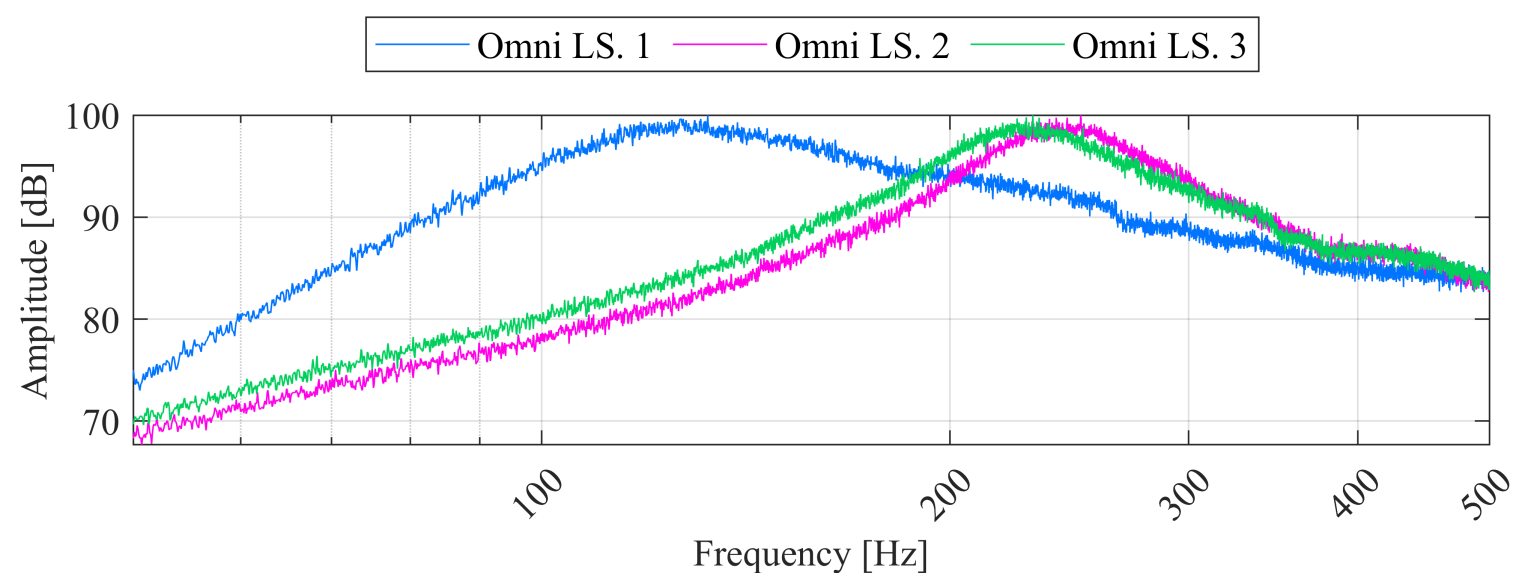

Figure 4: Normalized data from loudspeaker characteristic measurements in an anechoic chamber. 
All three omnidirectional loudspeakers are dodecahedra. The first, Omni LS. 1, is commercial and has a diameter of $39 \mathrm{~cm}$. Omni LS. 2 is DTU made, and has a diameter of $28 \mathrm{~cm}$. Omni LS. 3 is commercial and has a diameter of $33.2 \mathrm{~cm}$. It is important to mention, that above $2 \mathrm{kHz}$ the loudspeakers are not omnidirectional. Figure 4 shows the normalized sound pressure level average over the four microphone positions whilst playing pink noise. The smaller loudspeakers (Omni LS. 2 and 3 ) show a top points around $240 \mathrm{~Hz}$ whereas Omni LS. 1 has a top point around $125 \mathrm{~Hz}$. It was concluded that the most smooth pink noise spectrum in the significant range (from $100 \mathrm{~Hz}$ ) is expected from Omni LS. 1 when choosing between the three loudspeakers.

\section{RESULTS AND DISCUSSION}

Measurements were carried out for the chosen omnidirectional loudspeaker and corner loudspeaker in chamber 904 with no volume diffusers. To ensure that the measured data is similar to that of the theoretical, it is compared in Figure 5. The priority in this section, is that most of the excitations below $100 \mathrm{~Hz}$ are similar as the modal overlap is lowest here. The peak removal method is also applied in these figures, but only on the corner and omnidirectional loudspeaker as the Green's function is assumed to have more exact peaks.

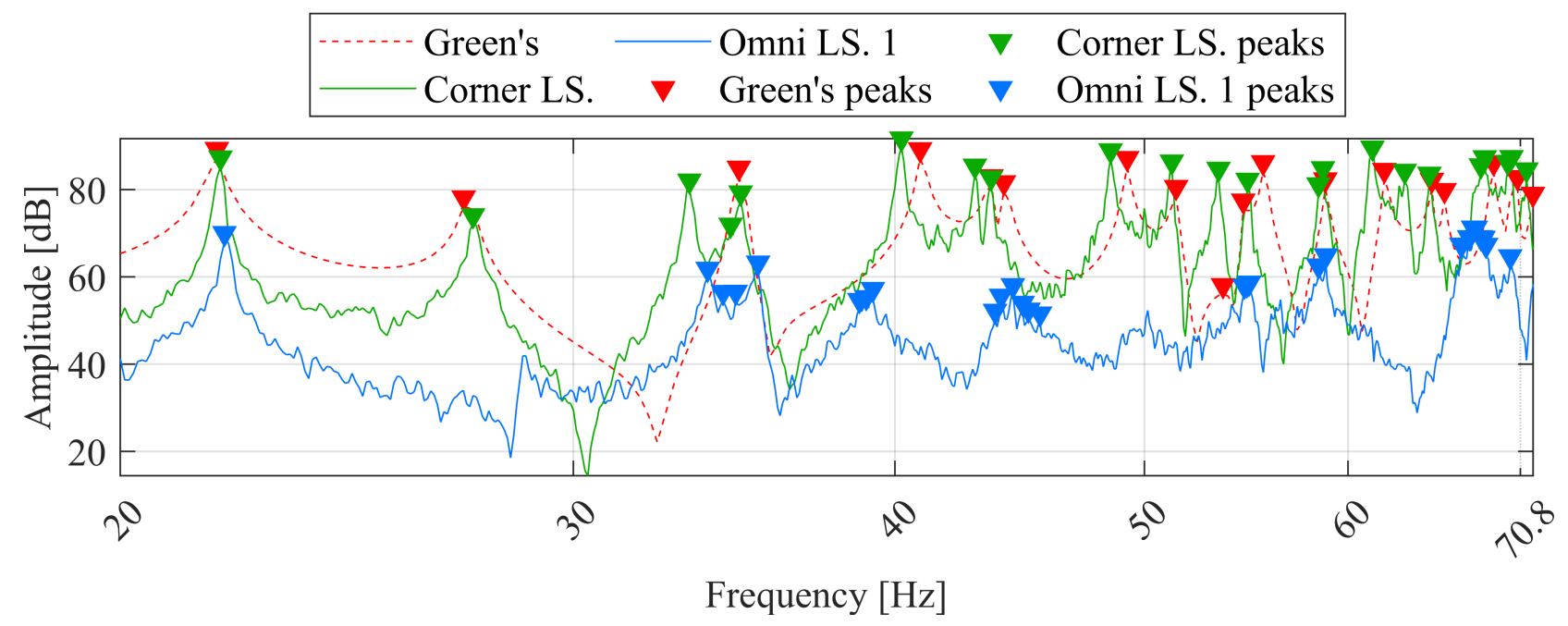

(a)

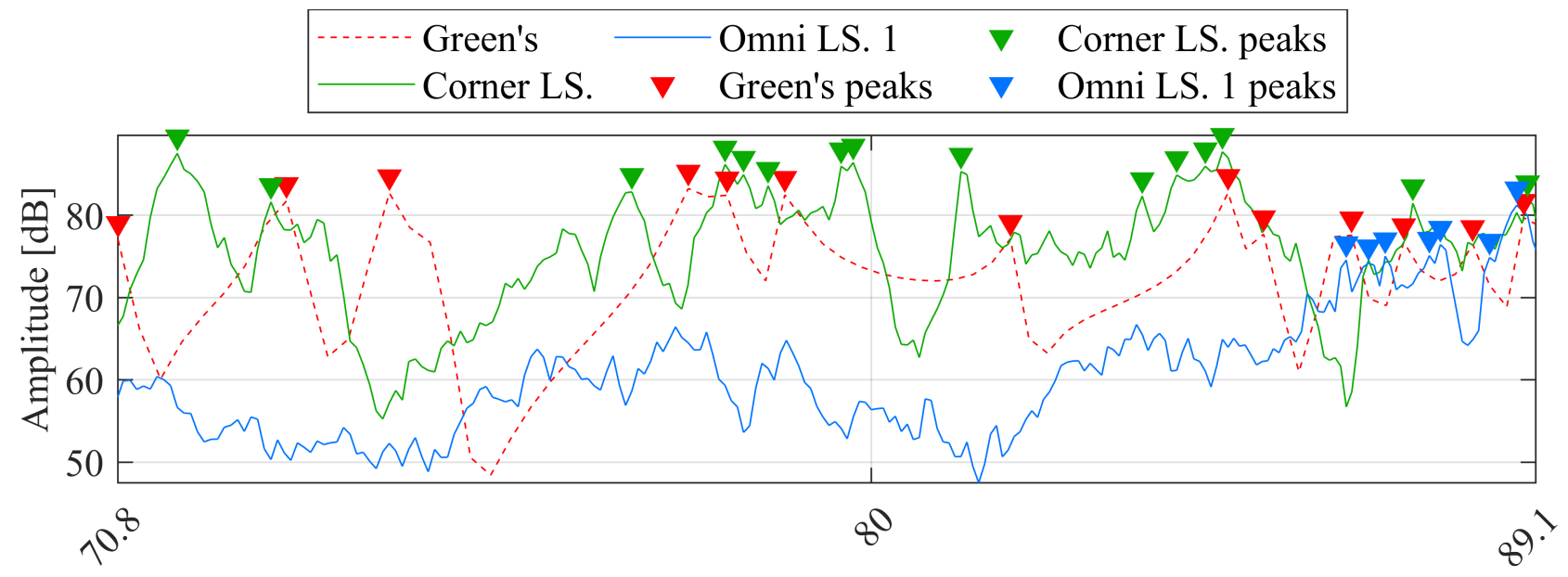

Frequency $[\mathrm{Hz}]$

(b)

Figure 5: Comparison of frequency response function in 904 in the intervals $20-70.8 \mathrm{~Hz}$ in subfigure (a), the $80 \mathrm{~Hz} 1 / 3$ octave band in subfigure (b) and the $100 \mathrm{~Hz} 1 / 3$ octave band in subfigure (c). 


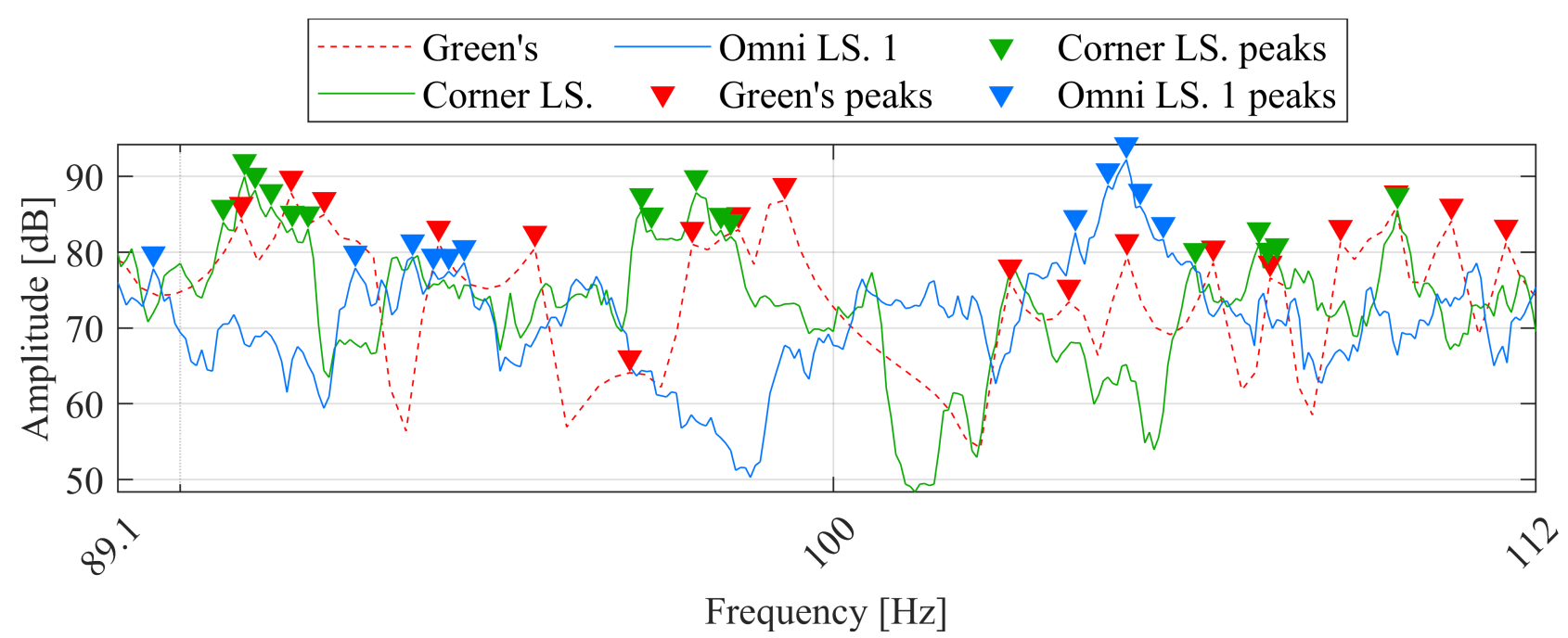

(c)

Figure 5: Comparison of frequency response function in 904 in the intervals $20-70.8 \mathrm{~Hz}$ in subfigure (a), the $80 \mathrm{~Hz} 1 / 3$ octave band in subfigure (b) and the $100 \mathrm{~Hz} 1 / 3$ octave band in subfigure (c). (cont.)

As seen in Figure 5 the frequency response function of the Green's function and the corner loudspeaker are most alike, but the omnidirectional loudspeaker also follows a very similar shape although its excitations are not sufficiently loud. As the excitations are not as great, this could cause problems in the peak detection due to a lower energy average level and thus leading to a larger peak count. The same problem does not persist for the corner loudspeaker just below the $80 \mathrm{~Hz} 1 / 3$ octave band. When comparing the Omni LS. 1 loudspeaker to the Green's function in Figure 5b, the omnidirectional loudspeaker also has trouble getting excitations at the same frequencies which could allude to the loudspeaker not being powerful enough. This might be due to the acoustic center of Omni LS. 1 not coinciding with the source point in the Green's function. For the $100 \mathrm{~Hz} 1 / 3$ octave band in Figure 5c, Omni LS. 1 appears to achieve several peaks at levels with Corner LS. This could be due to the pattern seen in Figure 2 where Omni LS. 1 peaks at about $125 \mathrm{~Hz}$. Both loudspeakers appear to have issues achieving results similar to the Green's function above the $100 \mathrm{~Hz}$ band, although achieving some similar peaks.

A comparison of the number of modes found at which frequency, is shown in Figure 6.

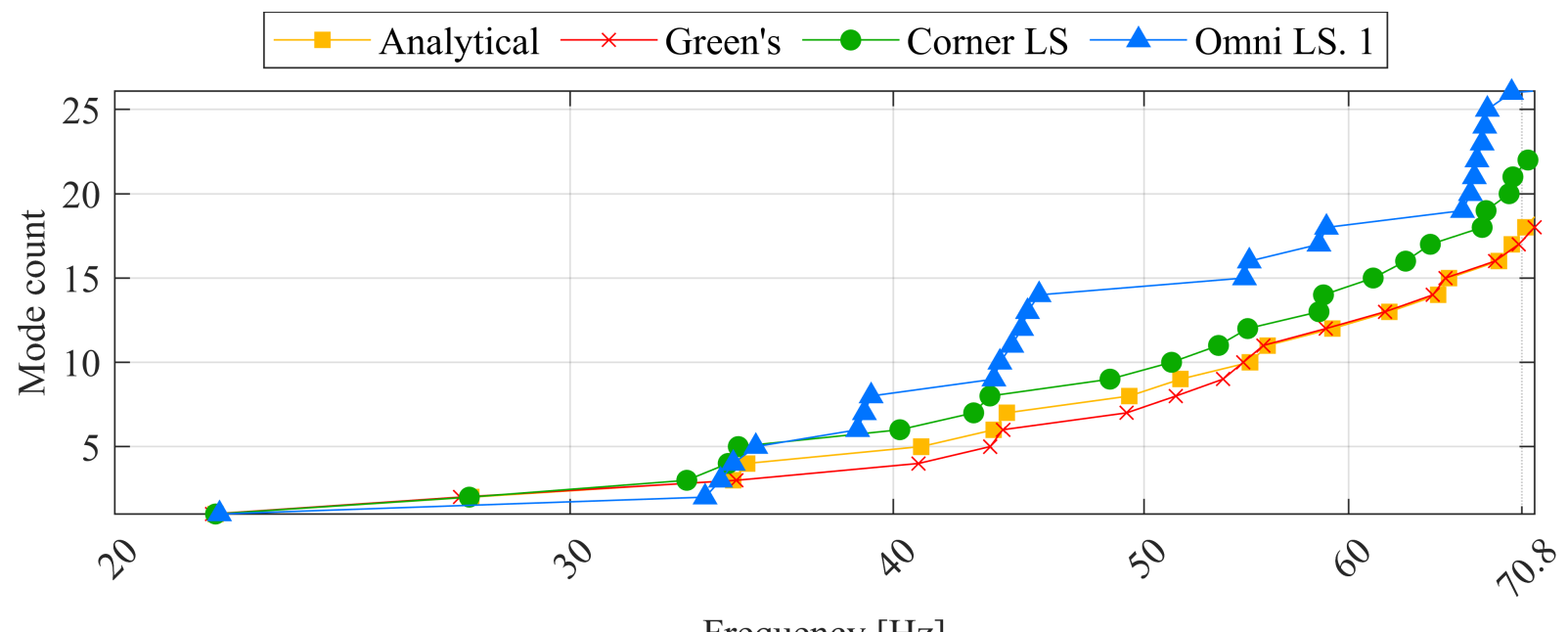

Frequency $[\mathrm{Hz}]$

(a)

Figure 6: Room mode count comparison in 904 in the intervals $20-70.8 \mathrm{~Hz}$ and the $70.8-447 \mathrm{~Hz}$. Both loudspeakers are mounted in the corner of the room, with microphone at the opposite wall. 


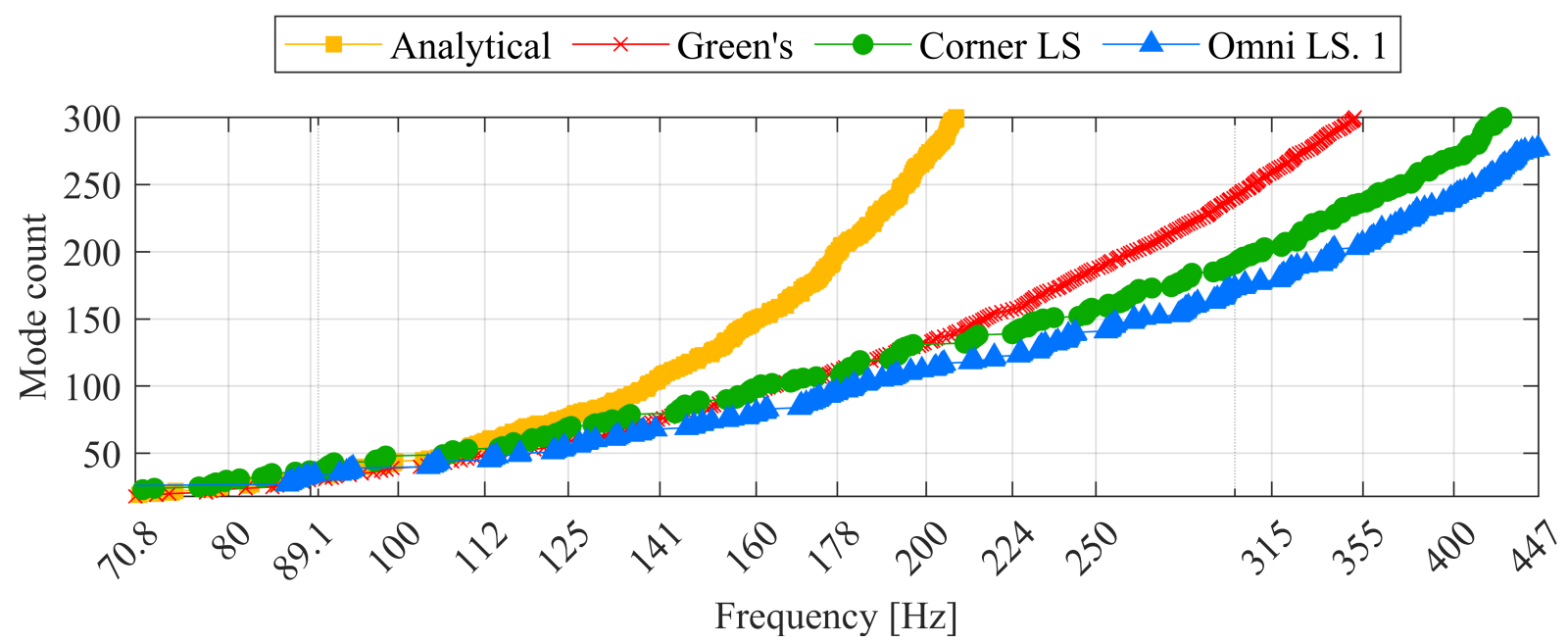

(b)

Figure 6: Room mode count comparison in 904 in intervals $20-70.8 \mathrm{~Hz}$ and the $70.8-447 \mathrm{~Hz}$. Both loudspeakers are mounted in the corner of the room, with microphone at the opposite wall. (cont.)

In Figure 6a the omnidirectional loudspeaker misses one of the first room modes and subsequently counts a much larger amount of room modes than the other three, mainly due to five peaks being located at about $45 \mathrm{~Hz}$. Although the corner loudspeaker also counts a larger number, its count is much closer to both the analytical and Green's function. This persists in Figure $6 \mathrm{~b}$ where it is clear that the analytical mode count becomes more dense, making it incomparable from $125 \mathrm{~Hz}$. In the final part, the Green's function count exceeds the count of both the corner- and omnidirectional loudspeaker. This happens at about $200 \mathrm{~Hz}, 150 \mathrm{~Hz}$ below the Schroeder frequency which is about $350 \mathrm{~Hz}$ for this reverberation chamber. It was however, expected that the counting procedure would become increasingly invalid as the modal overlap grows thus it would become very difficult to locate the exact mode.

To compare the counts, a bar chart is shown in Figure 7. The room mode excitation count is compared with the number of peaks found in the Green's function. It is not compared with the analytical count, as this would become invalid after $125 \mathrm{~Hz}$ as shown in Figure $6 \mathrm{~b}$. The number of room modes counted in the $1 / 3$ octave band is displayed on the top of the respective bars.

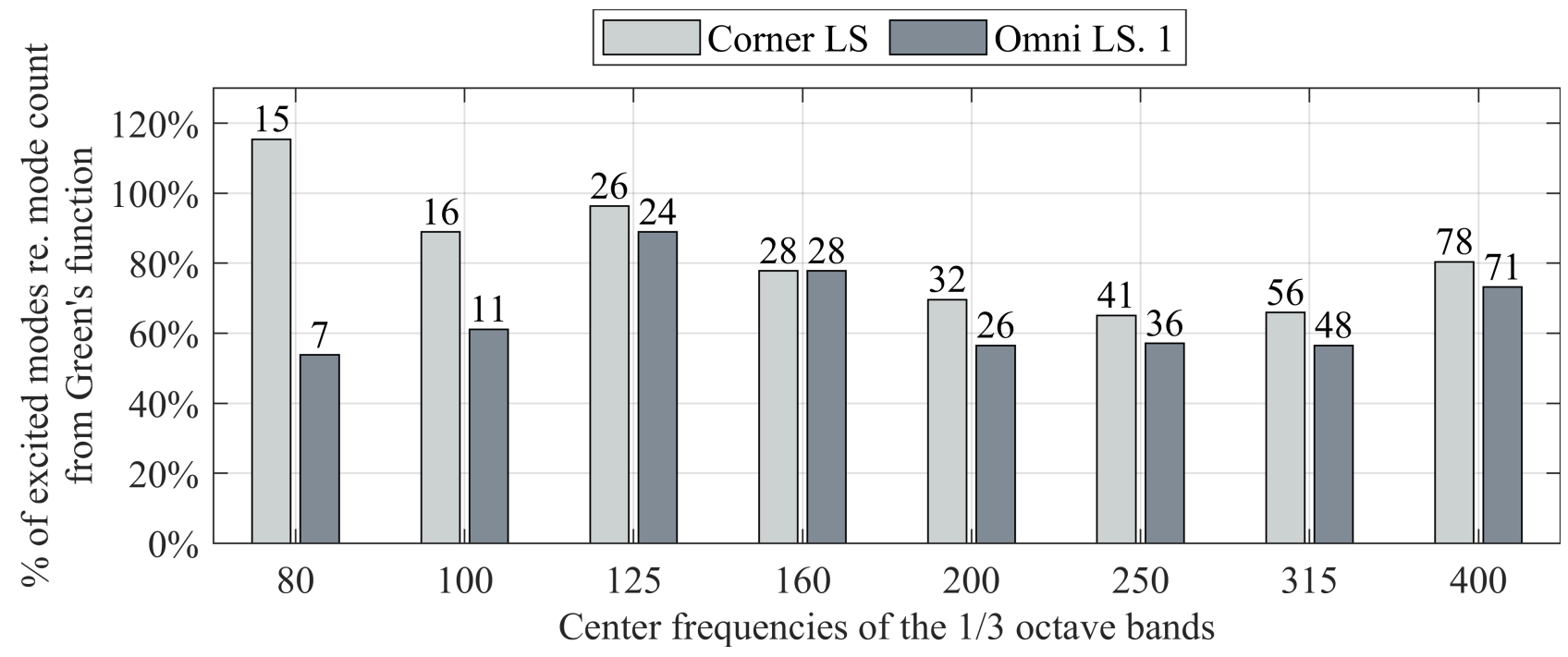

Figure 7: Room mode count in 904 
Initially, an interesting thing to note from Figure 7 is the increased peak count in the $80 \mathrm{~Hz} 1 / 3$ octave band. This is likely due to the measurement having multiple peaks around one room mode as in Figure $5 \mathrm{~b}$ just below $80 \mathrm{~Hz}$. As a almost $20 \%$ increased count is only two peaks in Figure 7 it is not considered a problematic factor. The omnidirectional loudspeaker appears to have a consistent problem in the 80 - and $100 \mathrm{~Hz} 1 / 3$-octave band, falling far behind on the count of peaks, then obtaining a more accurate count for the following two 1/3-octave bands. Both loudspeakers seem to fall far behind on the count in individual octave bands above the $200 \mathrm{~Hz} 1 / 3$-octave band, likely due to the modal overlap becoming above one at about $220 \mathrm{~Hz}$.

\subsection{Procedure example in non-rectangular chamber}

To investigate the procedures effect in a non-rectangular room, it is tested for three loudspeaker locations in chamber 005. The omnidirectional loudspeaker is placed at a corner, edge and in the center of the room to investigate which position would achieve the best results. Loudspeaker coordinates are corner $(7.44,5.90,0.20)$, edge $(7.44,5.90,0.72)$ and center $(3.92,3.23,0.92)$. The microphone is placed at an edge at $(0.05,3.45,0.25)$. In theory, the largest amount of room mode excitations should be achieved by placing the loudspeaker in a corner, thus this is the expectation with the position in the center of the chamber achieving the lowest amount of excitations. The results are not expected to be as precise as in chamber 904 because Green's function is valid for a rectangular room without diffusing elements. As the three positions have different Green's functions, they are compared separately to each respective Green's function as shown in Figure 8.

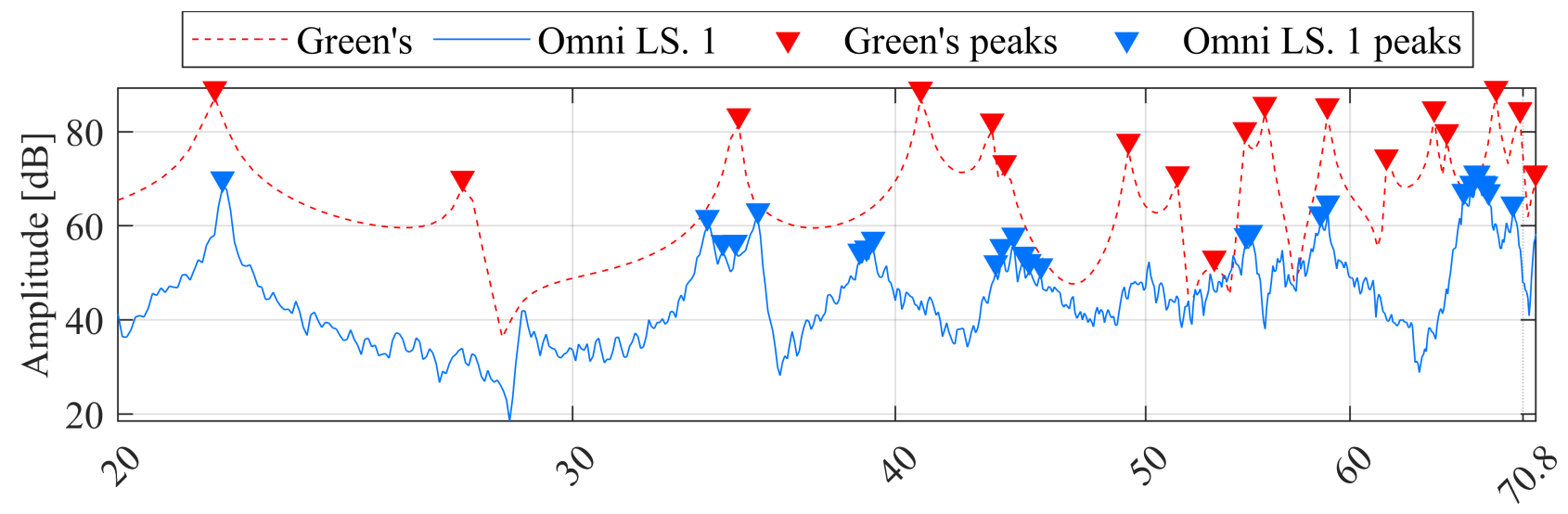

Frequency $[\mathrm{Hz}]$

(a)

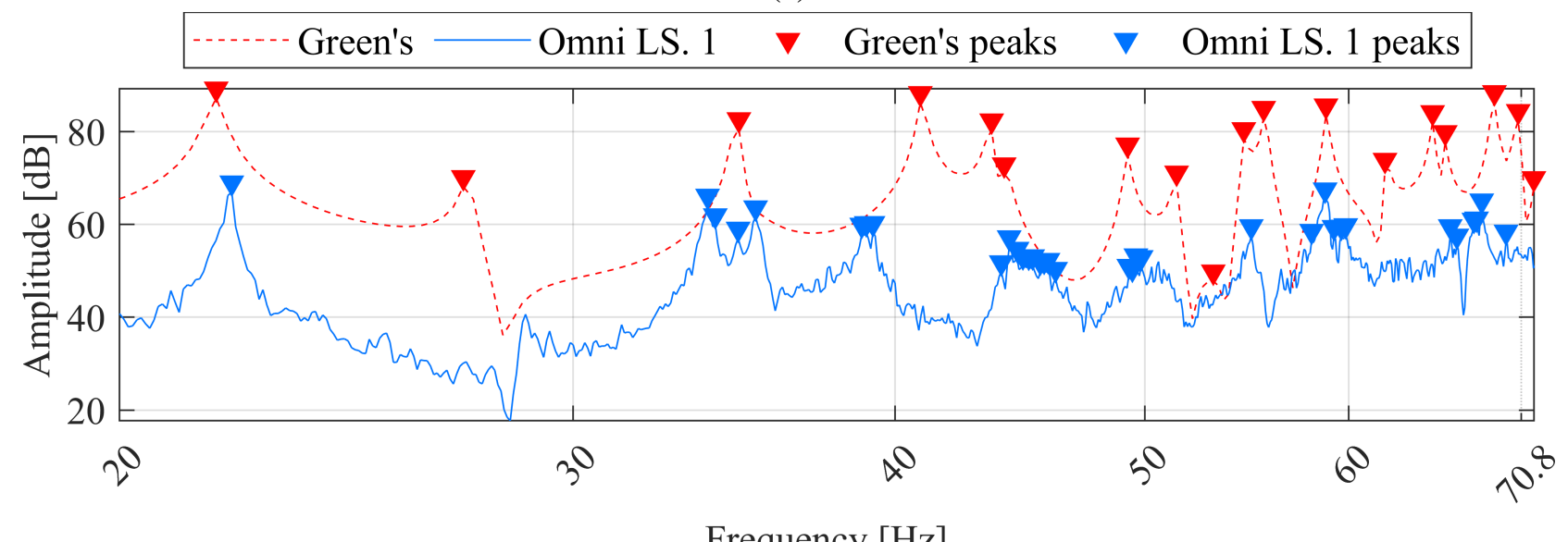

(b)

Figure 8: Omni LS. 1 compared to Green's function in 005 at corresponding measurement locations. Subfigure (a) is corner position, subfigure (b) is edge position and subfigure(c) is center position. 


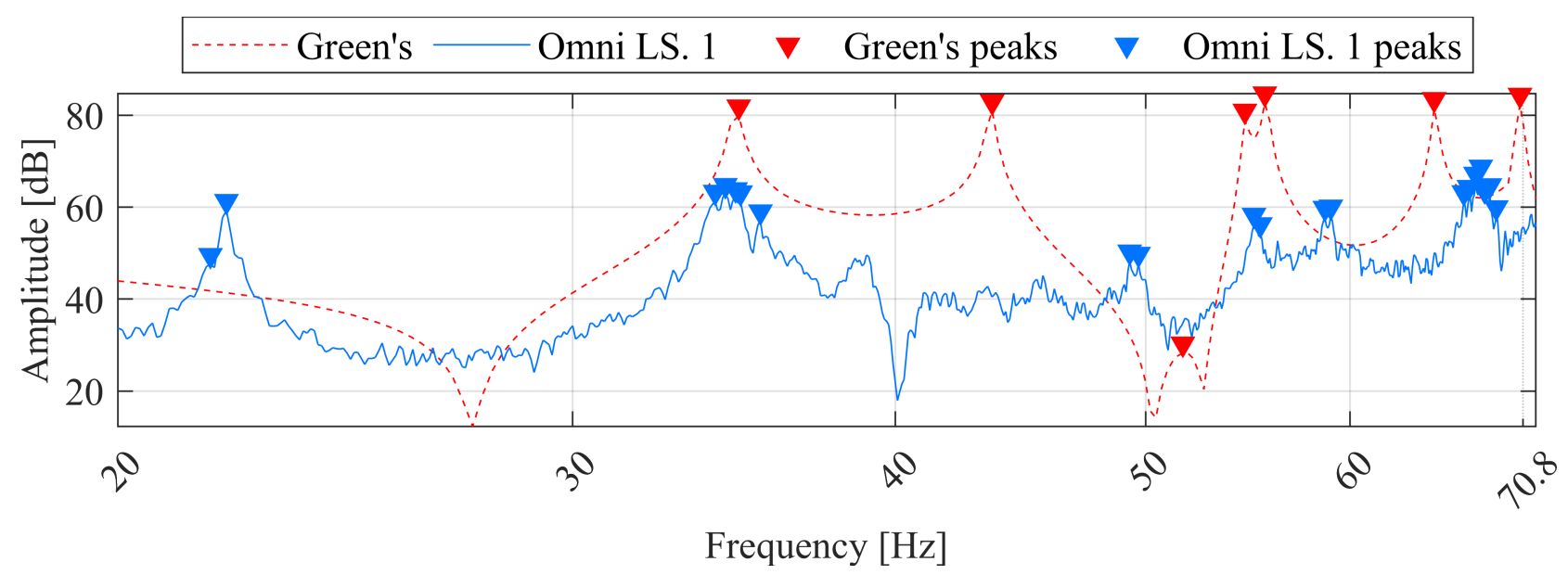

(c)

Figure 8: Omni LS. 1 compared to Green's function in 005 at the corresponding measurement locations. Subfigure (a) shows corner position, subfigure (b) shows edge position and subfigure(c) shows center position.(cont.)

In Figure 8a for the comparison of Green's function and the measurement, several peaks are almost aligned, though the general shape of the frequency response function of the measurement does not follow that of Green's function, especially just below the $80 \mathrm{~Hz} 1 / 3$ octave band. For the edge position in Figure 8b the Green's function curve is similar to that of the corner position, but the measurements frequency response function has altered slightly, primarily above $60 \mathrm{~Hz}$ causing it to deviate. Several more peaks are also detected by the procedure. In Figure $8 \mathrm{c}$ both curves have changed dramatically, producing much fewer peaks. Both results were expected, due to the loudspeaker position and the complexity of the chamber. From the three positions in Figure 8 the edge and corner position indicate to be favorable positions.

The count of room modes are shown in Figure 9 to study how the number of room modes follow the Green's function and analytical count of room modes.

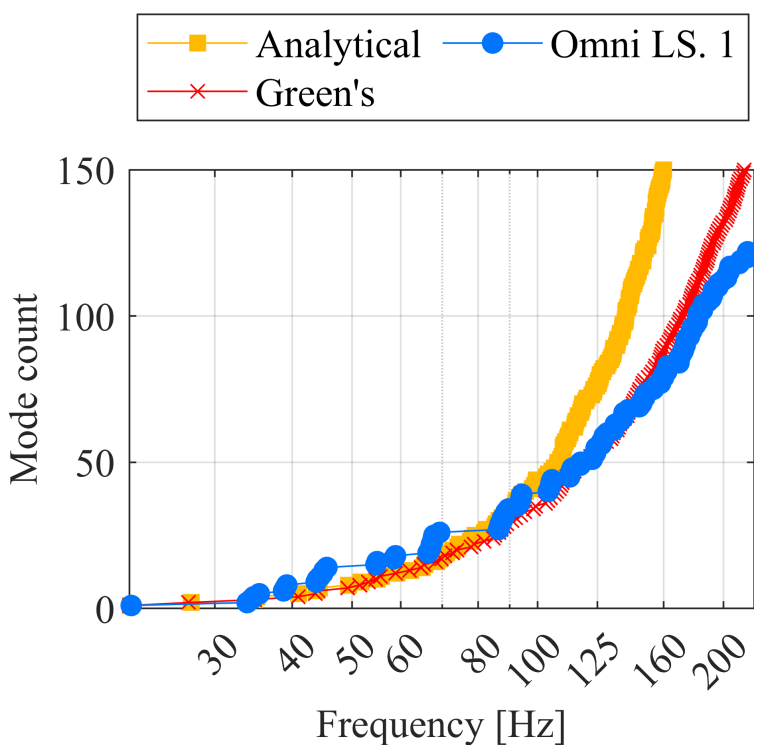

(a)

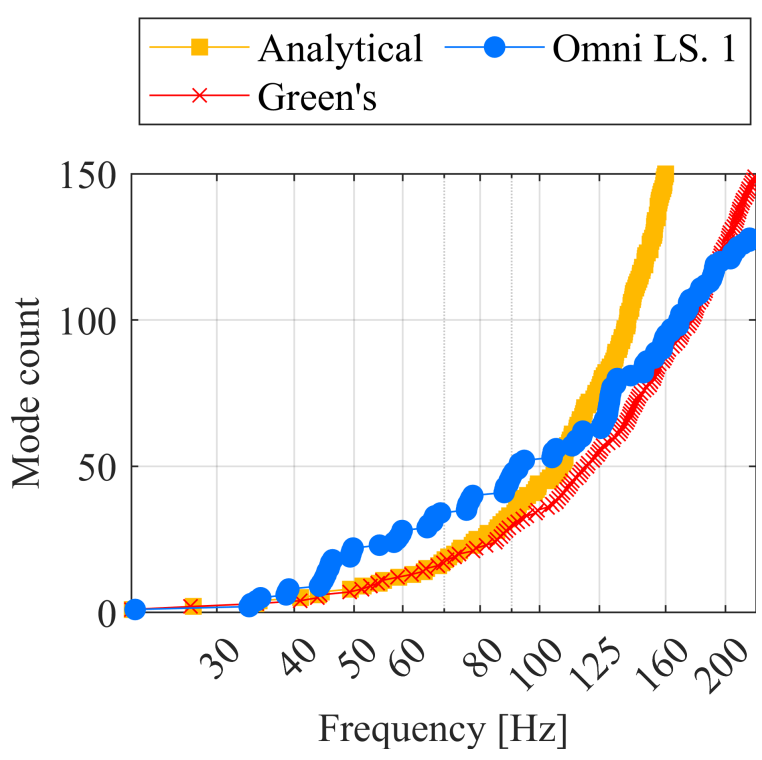

(b)

Figure 9: Room mode count of Omni LS. 1, Green's and analytical in 005. Subfigure (a) shows corner position, subfigure (b) shows edge position and subfigure(c) shows center position. 

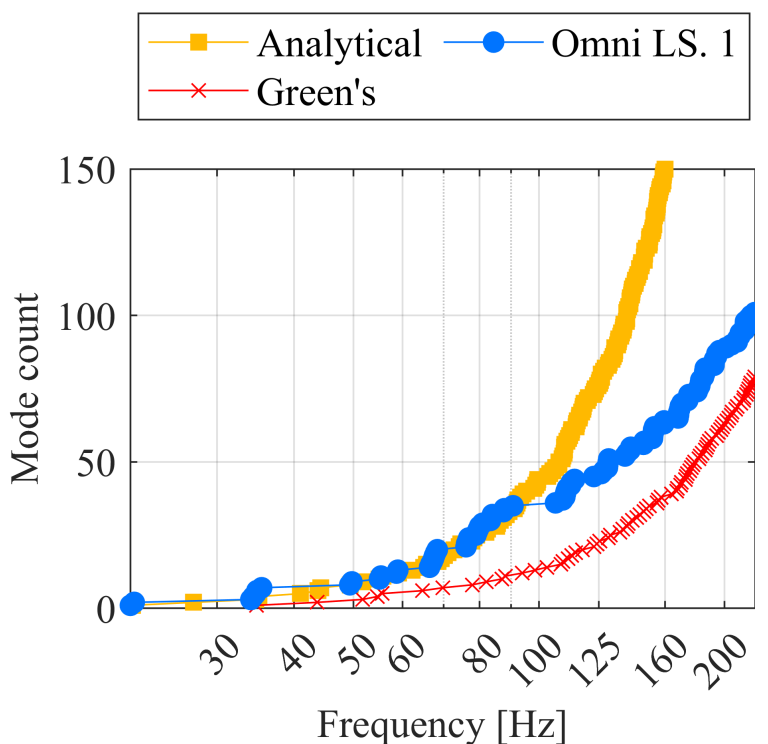

(c)

Figure 9: Room mode count of Omni LS. 1, Green's and analytical in 005. Subfigure (a) shows corner position, subfigure (b) shows edge position and subfigure(c) shows center position (cont.)

From Figure 9a it can be seen that the count closely follows the Green's function count to about $141 \mathrm{~Hz}$ where it starts to fall behind. In Figure $9 \mathrm{~b}$ the count exceeds the Green's function below the $200 \mathrm{~Hz} 1 / 3$-octave band and falls behind in the following $1 / 3$-octave band. For the center position in Figure 9c, the count exceeds that of the Greens function all the way through, following the count of the analytical amount of room modes more closely till the end of the $80 \mathrm{~Hz} 1 / 3$-octave band. From these three figures, considering the Green's function a "perfect situation" would make the corner position most favorable. If instead the maximum number of peaks is preferred, the edge position in Figure $9 \mathrm{~b}$ appears to achieve a slightly higher amount than the corner position.

Finally the number of modes counted in $1 / 3$ octave bands from the $80 \mathrm{~Hz}$ band to the $400 \mathrm{~Hz}$ band is compared in Figure 10.

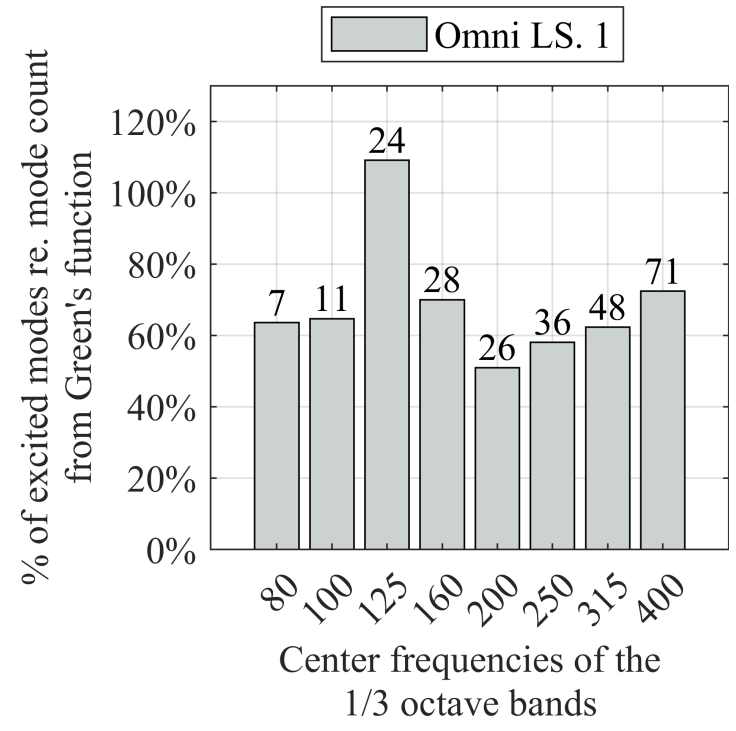

(a)

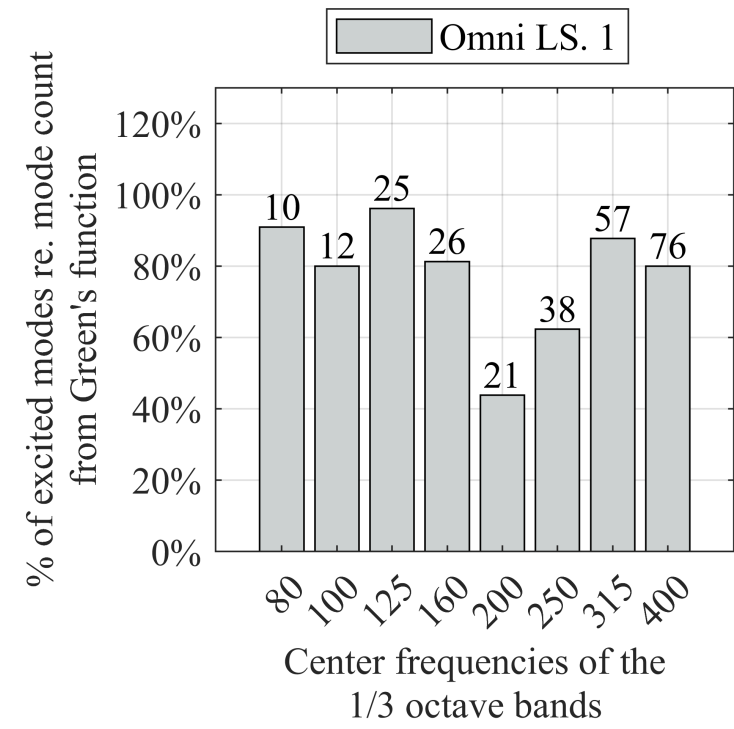

(b)

Figure 10: Room mode count in 1/3-octave bands compared to Green's function in 005. Subfigure (a) shows corner position, subfigure (b) shows edge position and subfigure(c) shows center position. 


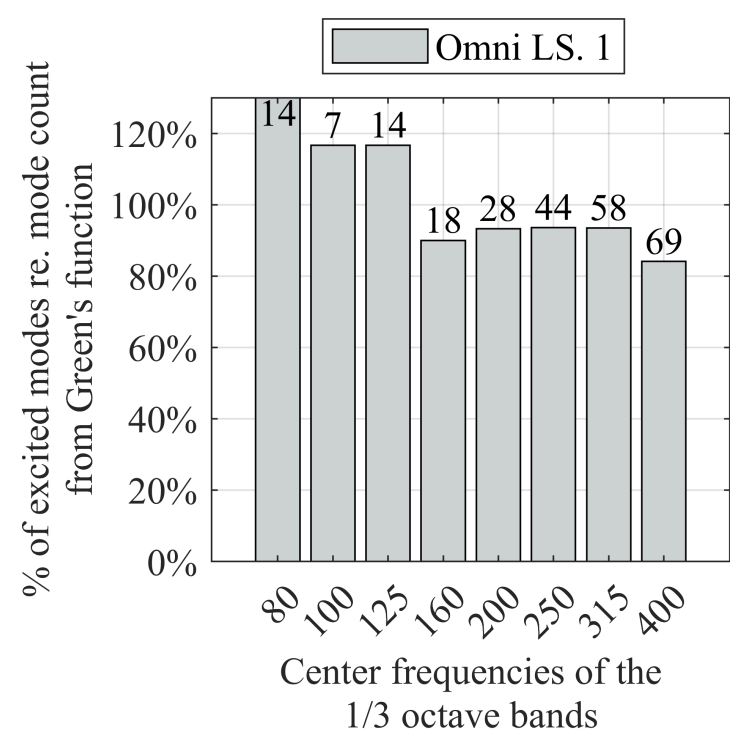

(c)

Figure 10: Room mode count in 1/3-octave bands compared to Green's function in 005. Subfigure (a) shows corner position, subfigure (b) shows edge position and subfigure(c) shows center position. (cont.)

The number of room modes in $1 / 3$-octave bands in Figure 10a shows a low count compared to that of the Green's function which is interesting as the count appeared to follow a similar curve in Figure 9a. For the edge position in Figure $10 \mathrm{~b}$ the count is more precise compared to the count of Green's function in the majority of the shown $1 / 3$ octave bands. For the center position in Figure $10 \mathrm{c}$, the count exceeds greatly in the $80 \mathrm{~Hz} 1 / 3$ octave band (to $350 \%$, not shown on figure), but the rest are almost the same as for the Green's function. The exceeded count could be caused by what happens below the $80 \mathrm{~Hz}$ octave band in Figure 8c, where several peaks appear at what is likely just one room mode excitation. Although the \% count is very close to the Green's function, Figure $8 \mathrm{c}$ is misleading as the Greens function misses any mode that has nodal lines at the center of the chamber, whilst this is not the case for the experiments due to the complex shape of the chamber.

The investigation of the complex shaped chamber indicates that either the corner or edge position is most favorable, as these positions excite most peaks in measurements and Green's function. As this example has primarily studied the low frequencies, a final conclusion on which position is most favorable is not given. As the Green's function frequency response function is not very similar to the measurements, it is recommended that further research is done for the method to include complex shaped reverberation chambers.

\section{SUGGESTED METHOD FOR DETERMINING A LOUDSPEAKER LOCATION}

On the basis of the studies in this paper, a method for determining preferable loudspeaker locations is devised as the following:

1. Analyze Green's function for relevant loudspeaker and microphone position combinations

2. Conduct steady state measurements to obtain frequency response function for favorable positions

3. Remove peaks using proposed removal method

4. Count remaining peaks 
5. Evaluate peak percentage, number and the average level of the curve.

It is important to remember to not only look at the number or \% of peaks compared to the Green's function, as the average energy level may be low enough to include several peaks which may not be room modes. The results may take a bit of time to look through, but it may be worth it to obtain the best possible measurements.

\section{CONCLUSION}

A procedure for locating favorable loudspeaker locations has been proposed. The investigation has used optimal situations such as a rectangular reverberation chamber and optimal loudspeaker choice to study the efficiency of the procedure. The procedure would need future work to be more efficient, but is in its current state applicable. As omnidirectional loudspeakers may not perform as well as corner loudspeakers in the lower frequencies, it is suggested that certain requirements are made for the usage of omnidirectional loudspeakers in such measurements.

For rectangular reverberation chambers which do not include large elements e.g. volume diffusers, the procedure can be used. It is, however, important to note that once the modal overlap exceeds 1 , the procedure appears to fall behind on room mode count. Further research into precise location of room modes is recommended, especially at frequencies where only one mode is supposed to occur, but several more are counted.

As the procedure did not show very precise results for the complex shaped chamber, it is not recommended for use in such chambers yet. Further research would be suggested, as to how frequency response curves could be simulated for such chambers.

\section{ACKNOWLEDGEMENTS}

Thanks to Rasmus Stahlfest Holck Skov from FORCE Technology for guidance on measurement and equipment setup.

\section{REFERENCES}

[1] P. Lord Z. Maekawa, J. H. Rindel. Environmental and Architechtural Acoustics 2nd Edition. Spon Press, 2011.

[2] Martijn Vercammen. On the revision of iso 354, measurement of the sound absorption in the reverberation room. In Janina Fels Martin Ochmann, Michael Vorländer, editor, Proceedings of the 23rd International Congress of Acoustics, pages 3991-3997. Deutsche Gesellschaft für Akustik e.V.(DEGA), September 2019.

[3] Cheol-Ho Jeong, Gerd Marbjerg, and Jonas Brunskog. Uncertainty of input data for room acoustic simulations. In Proceedings of bi-annual Baltic-Nordic Acoustic Meeting, 2016. Bi-annual Baltic-Nordic Acoustic Meeting 2016, BNAM 2016 ; Conference date: 20-06-2016 Through 22-06-2016.

[4] Peter M. Juhl Finn Jacobsen. Fundamentals of General Linear Acoustics. John Wiley \& Sons Ltd, 2013.

[5] Niels Peter Moos. Loudspeaker configuration for iso 354. Bachelor's thesis, Technical University of Denmark, January 2021. 